\title{
Infrared Emission from Barred Spiral Galaxies
}

\author{
Tim G. Hawarden ${ }^{1}$ \\ Joint Astronomy Centre, 660 N. A'ohoku Place, Hilo, Hawaii 96720, \\ $U S A$
}

J. H. Huang and Q. S. Gu

Astronomy Department, Nanking University, Nanking, China

\begin{abstract}
Amongst relatively undisturbed spiral galaxies of type $\leq$ Sc barred morphology is unquestionably associated with powerful mid-and Far-IR emission. On the other hand, even amongst early type galaxies, those with $L_{F I R} / L_{B}<1 / 3$ exhibit no association of high relative FIR luminosity with barred morphology, but some association of IR colors resembling those of star formation regions (SFRs). Amongst systems with $L_{F I R} / L_{B}<0.1$ this ratio may be anti-correlated with barredness. It appears that enhanced IR emission from those galaxies whose star formation rate is currently elevated by the the bar translates them into the group with higher FIR-to-optical luminosity ratios. Depletion of extended nearnuclear gas and dust, once the bar has swept up the currently-available supplies, may reduce the fraction of the background stellar radiation field which can be converted to FIR radiation in the inner, most luminous parts of the galaxy. Thus, after the starburst has subsided, such galaxies may be less FIR-luminous than unbarred systems. Several uncertainties remain: it is still not clear whether barred morphology is a necessary condition for the generation of a starburst in an otherwise undisturbed galaxy, while evidence as to the effect of differing bar strengths is conflicting.
\end{abstract}

\section{Introduction}

One of the first indications that the nuclei of barred spiral galaxies harbor structures unlike those of non-barred systems was provided in papers by Sérsic \& Pastoriza $(1965,1967)$ listing spiral galaxies with "peculiar" bright nuclear structures, often clearly-delineated ("hot-spot") complexes, apparently of HII regions. All these galaxies were classified SB or SAB in the Reference Catalogue of Bright Galaxies (de Vaucouleurs \& de Vaucouleurs 1964) apart from NGC 1097, an obvious misprint in the 1967 paper.

Galaxy models have long suggested that bars are effective mechanisms for moving the ISM in the inner parts of a galaxy to smaller radii. Roberts, Huntley

${ }^{1}$ and Royal Observatory, Blackford Hill, Edinburgh EH9 3HJ, UK 
\& van Albada (1979) showed that the velocity field of the gaseous ISM inside the radius of a bar exhibits a definite net inward component. Tubbs (1982) and Schwarz (1984), amongst others in that decade, showed that the effects of shocks can be to move material inwards in quite significant quantities. Athanassoula $(1992 \mathrm{a}, \mathrm{b})$ provides excellent reviews - with extensive references to earlier work - of the processes involved, which obviously have the potential to concentrate near the nucleus the raw material for an outburst of star formation.

\section{Early Observations of IR Emission From Barred Spirals}

Quantifying the luminosity of a SFR must be done in the IR. Telesco \& Gatley (1981) showed that the spectacular ring of HII regions surrounding the nucleus of the strongly barred galaxy NGC 1097 is a powerful source of IR emission from warm dust heated by young stars. In a subsequent study (1984) they observed similar emission from the circumnuclear ring in the SAB galaxy NGC 3310 and showed that by any definition this complex comprises a "starburst", since starformation has only recently commenced, while the star formation rate inferred from the IR emission can by no means be maintained indefinitely.

\section{The IRAS Survey: de Jong et al. 1984 and Hawarden et al. 1986}

IRAS provided, for the first time, extensive information on the mid- and far-IR properties of galaxies of all classes. Thus de Jong et al. (1984) found that 108 out of 165 RSA galaxies (Sandage \& Tammann 1981) were detected by IRAS. Amongst early-type spirals the barred systems had a relatively higher detection rate than unbarred systems, and barred systems tended to have higher ratios of 60 to $100 \mu \mathrm{m}$ emission and of $L_{F I R} / L_{B}$.

Hawarden et al. (1986a,b) noted that known starburst galaxies like NGC 1097, NGC 3310 and M82 had IR spectral energy distributions (SEDs) like those of compact galactic HII regions, rising rapidly from 12 to $25 \mu \mathrm{m}$ and slowly from 25 or $60 \mu \mathrm{m}$ to $100 \mu \mathrm{m}$, while those of "normal" galaxies (e.g. NGC 5033: Hawarden et al. 1986a, Figure 2) are nearly flat between 12 and $25 \mu \mathrm{m}$ and rise more steeply to longer wavelengths. They accordingly used the "25/12" ratio (of the mid-IR fluxes observed by IRAS at $25 \mu \mathrm{m}$ and at $12 \mu \mathrm{m}$ ) and the somewhat less sensitive 100/25 ratio as indicators of star formation activity in a two-color diagram. On a plot of galaxies from the RSA classified SA, SAB or SB in the RC2 (de Vaucouleurs et al. 1976), with detections in all four IRAS bands, the barred and unbarred objects have very different distributions: the area associated with high star formation rates $(25 / 12>2.2)$ contains a total of 37 galaxies, of which 23 are classified SB, $12 \mathrm{SAB}$, and only $2 \mathrm{SA}$. The mean $L_{F I R}$ of the barred galaxies from the sample is about twice that of the unbarred systems.

Hawarden et al. (1986b) therefore asserted that the presence of a bar is strongly associated with, and very likely causes, enhanced star formation in non-interacting, non-merger systems, and might well be a necessary condition. 


\section{Confirmation and Amplification}

\subsection{Bars, IR-brightness and Early Morphological Types: Devereux et al. 1987 and Devereux 1987}

Devereux, Becklin \& Scoville (1987) discussed the near- and mid-IR properties of a volume-limited sample of nearby galaxies. They did not discern significant dependence of (ground-based or IRAS) mid- and far-IR properties on barred morphology, but supported and emphasized a suggestion by Hawarden et al. (1986b): more explicitly, they asserted that the effects of barred morphology are important only for intense star-formation (episodes) and not for the steadystate, lower-level star formation in "normal" systems.

This was soon strongly confirmed by Devereux (1987) who studied a larger sample, containing numerous IR-luminous spiral systems. Like Hawarden et al. (1986b), he found a strong correlation of barred morphology with IR colors, but also with the degree of central concentration of mid-IR $(10 \mu \mathrm{m})$ emission. These effects were apparent only for early-type galaxies ( $\mathrm{T}<5$ or so).

\subsection{Confirmation of Bar Effects}

Dressel (1988) found that galaxies with high $60 / 100$ ratios (indicating warmer average dust temperatures) were predominantly barred, and confirmed that the effects were most apparent in early-type systems, extending even to SO galaxies, despite their canonically low star-formation rates. Puxley, Hawarden \& Mountain (1988) segregated barred galaxies into "high" and "low" groups based on their 25/12 ratios ( $>$ or $<2.2$ respectively) and, following Hummel (1981), showed that concentrated $\left(<20^{\prime \prime}\right)$ central radio sources are much more common in the former - presumably actively star-forming - systems than in the latter, and even less common amongst unbarred galaxies. Rowan-Robinson \& Crawford (1989) modeled the IRAS colors of galaxies as a combination of AGN, "Starburst" and "disc" components. They found that barred systems showed a marked tendency to contain a higher "starburst" fraction than unbarred galaxies.

\section{Controversy}

\subsection{Are Bars Really Needed? Pompea \& Rieke 1990}

The strong assertions of Hawarden et al. in (1986a,b) did not continue unchallenged, however. In 1990 Pompea \& Rieke published a study of spirals chosen for a probable high relative level of star-forming activity (high IRAS 60/100 ratio, $\left.L_{F I R}>10^{10} L_{\odot}\right)$, but excluding those appearing on the sky survey plates to be interacting, merging or barred. They secured $K$-band images of 15 of these galaxies which were then analyzed for oval and bar distortions which might have been hidden or diluted by extinction effects at visible wavelengths. They concluded that out of the 10 galaxies in their selection which showed $25 \mu \mathrm{m}$ excesses by the criteria of Hawarden et al. (1986b) only three were barred, while out of the original sample of 22 IR-selected "non-interacting" galaxies at least 8 "do not have strong bars", apparently contra Hawarden et al. (1986b). 
Some of the reclassifications will startle optical morphologists, including descriptions of the "bars" in NGC 253 and NGC 3310 as spiral arms emerging from a nucleus. Amongst other listed examples of "unbarred" star-forming systems are NGC 922 and NGC 5936, which are classified SB in the RC2. Overall, although the optical morphological types listed in the paper do not show any of the 15 objects as barred, the RC2 categorizes 3 as SBs and 10 as SABs. Two are unclassified and only one is an SA (Huang et al. 1995); all the systems with $25 / 12>2.2$ are classified $\mathrm{SB}$ or $\mathrm{SAB}$ in the $\mathrm{RC} 2$. Irrespective of the outcome of the NIR reclassification by Pompea \& Rieke, the RC2 classification of their sample galaxies fully supports the assertions by Hawarden et al. (1986a,b). Evidently, whatever property led a galaxy to be classified as $S B$ or SAB in the RC2 is strongly associated with high 25/12 ratios, high IR luminosities and probably high star formation rates. We believe that this property actually is barredness, and that the NIR reclassifications are flawed.

\subsection{Beyond Argument: Young \& Devereux 1991}

These authors studied molecular clouds in an 18-galaxy sample previously selected as "starburst" systems, comprising all non-Seyfert 1 galaxies between 15 and $40 \mathrm{Mpc}$ distant, with central $10 \mu$ m luminosities $\geq 6 \times 10^{8} L_{\odot}, \delta>-20^{\circ}$, $22^{h}<\alpha<16^{h}$ and with $|\mathrm{b}| \geq 20^{\circ}$. The galaxies in this sample have mean $L_{F I R} / \mathrm{M}\left(\mathrm{H}_{2}\right)$ (a measure of the rate at which stars are being formed from gas) $\sim 11 L_{\odot} / M_{\odot}$, intermediate between that for a sample of "isolated" galaxies $\left(\sim 5 L_{\odot} / M_{\odot}\right)$ and that for interacting systems $\left(\sim 20 L_{\odot} / M_{\odot}\right)$. Clearly starforming activity in this "starburst" sample actually is enhanced.

Of the 18 galaxies, 10 are classified $\mathrm{SB}$ in the $\mathrm{RC} 2,7$ are classified $\mathrm{SAB}$, and once again only one (NGC 470) is an SA. Two probable post-merger systems are included: NGC 2146, classified SB(s)ab pec in the RC2, with its distorted morphology nicely shown in their Figure 1, and NGC 4194 (Arp 160), classified $\mathrm{IBm}$ pec in RC2. The rest of the galaxies in the list represent a reasonably normal sample of spiral galaxies chosen for IR-brightness, independent of morphology. Once more an IR-bright sample proves to be completely dominated by barred systems.

\subsection{Isobe \& Feigelson 1992: Bars Have Lower $L_{F I R}$ ?}

Isobe \& Feigelson (1992) carried out a statistical analysis of a volume-limited sample of nearby spirals, concluding that barred galaxies in their sample are actually about a factor two less luminous at FIR wavelengths than the unbarred systems.

Isobe \& Feigelson attribute the conflicting conclusions of the studies to differing sample selection criteria. Thus, the samples of Hawarden et al., while drawn from the optically-selected, and complete, RSA, are IR-selected in that they contain only systems detected by IRAS even at the difficult 12 and 25 $\mu \mathrm{m}$ bands, introducing a strong bias towards IR-luminous systems. Their own sample, on the other hand, being completely optically-selected, with (numerous) IR non-detections allowed for by survival analysis, has no such bias.

Isobe \& Feigelson suggest that an average SB galaxy is less luminous than an average SA system because of the effects of the bar in sweeping up the ISM in the central parts of the galaxy. When the starburst is no longer present, 


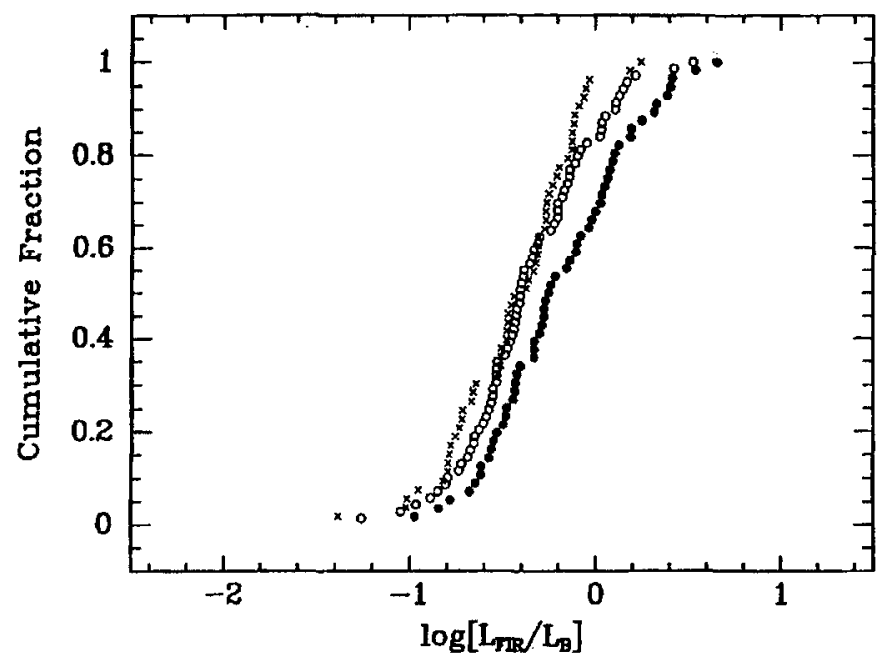

Figure 1. Cumulative distribution of $L_{F I R} / L_{B}$ for the IRAS Bright Galaxy Sample of Huang et al. (1995). Galaxies classified SA in the $\mathrm{RC} 2$ are shown as open circles, SAB systems as crosses and SB systems as filled circles. The SBs are markedly brighter in the IR, but the SABs are indistinguishable from the SAs.

the resulting paucity of dusty ISM means that less of the general interstellar radiation field (from the older stars) can be thermalized into the far-IR before escaping from the system completely.

\section{Resolution: Huang et al. 1995}

\subsection{The IR-Bright Samples: Bars Increase IR Emission...}

Huang et al. (1995) define an IR-bright "undisturbed starburst" sample from the IRAS Bright Galaxies sample of Soifer et al. (1989) (which is complete to IRAS $S_{60}=5.4$ Jy over a significant part of the sky) by eliminating AGNs listed by Véron-Cetty \& Véron (1993) and merging or interacting galaxies identified by Lonsdale, Persson \& Matthews (1984).

The cumulative frequency distribution of the $L_{F I R} / L_{B}$ ratio for this sample (Figure 1) clearly shows the SB systems to have about twice the relative IR luminosity of the SAs. However, puzzlingly, the SAB systems are indistinguishable from the SAs, in sharp contrast to the results of Hawarden et al. (1986b). This remains true even when the $25 / 12$ ratio, a more sensitive measure of IR properties than $L_{F I R} / L_{B}$, is used.

The new sample confirms that the effects of bars are confined to early-type systems and suggests that they become prominent only when $L_{F I R} / L_{B}$ exceeds $\sim 1 / 3$. 


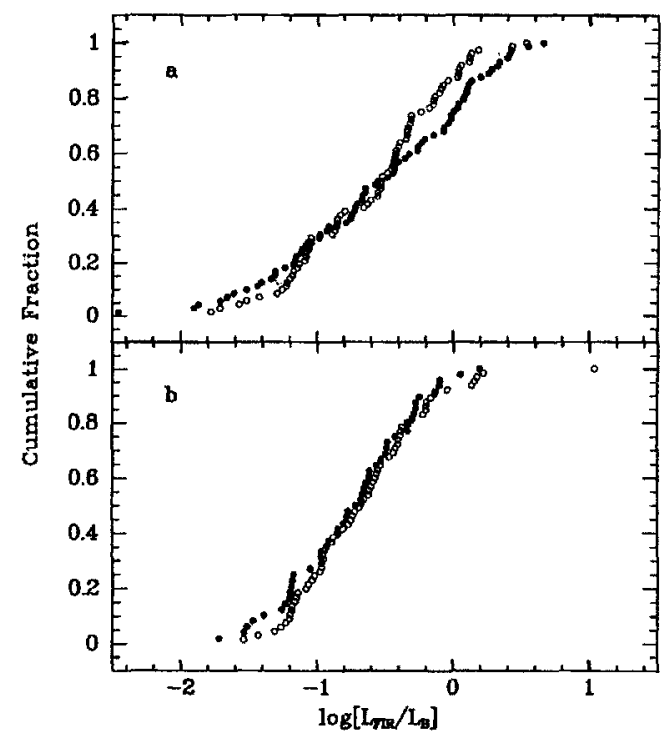

Figure 2. Cumulative distribution diagram of $L_{F I R} / L_{B}$ for the combined sample of Huang et al. (1995), with symbols as in Figure 1. (a) for early-type galaxies, (b) for types Sc and later. For $L_{F I R} / L_{B}>1 / 3$ the early-type barred systems are more luminous in the IR than the unbarred systems, but for $L_{F I R} / L_{B}<0.1$ the reverse is true.

\subsection{The IR-Faint Samples: Bars Decrease IR Emission...}

Huang et al. go on to examine an RSA-derived sample similar to those of Hawarden et al. (1986a,b). The majority of these galaxies have $L_{F I R} / L_{B}<1 / 3$ and in this sample the difference between the distributions of $L_{F I R} / L_{B}$ in barred and unbarred systems is small. Nevertheless there is a big difference between the distributions of the $25 / 12$ colors of the barred and unbarred galaxies. In addition to having a higher mean $25 / 12$ value the barred galaxies have a markedly broader distribution in color.

Huang et al. also re-examine the sample of Isobe and Feigelson (1992). This has mean $L_{F I R} / L_{B} \sim 11$ times lower than that for the IRAS Bright Galaxies and 2.3 times lower than the RSA-derived sample in the previous paragraph. The barred galaxies exhibit the lower $L_{F I R} / L_{B}$ relative to unbarred systems seen by Isobe \& Feigelson.

\section{Understanding the Mechanisms: Telesco, Dressel \& Wolstencroft 1993}

While we have convincing evidence that amongst IR-luminous early-type spiral galaxies the presence of a bar is associated with significant differences in IR 
properties, we have not discussed causes. This has been done by Telesco, Dressel $\&$ Wolstencroft (1993) as part of their analysis of mid-IR (mostly $10 \mu \mathrm{m}$ ) groundbased imaging of galaxies. Telesco et al. convincingly explain the variety of morphologies seen in the near-nuclear $10 \mu \mathrm{m}$ structures as a consequence of the inward sweeping effects of the bar on the ISM combined with accumulation and orbital perturbation at an inner Lindblad resonance (ILR) or near the nucleus itself, if an ILR is absent.

Telesco et al. find an impressive correlation of the likely presence or absence of an ILR with the presence of a ring-like, as opposed to a central, distribution of SFRs. This correlation is good enough to convince us that the basic problems of the genesis of starbursts, and thus of the IR emission from barred spiral galaxies, have been understood.

\section{Observational Overview of "Normal" Spiral Galaxies in the IR}

Two regimes of relative IR luminosity appear to exist, in which barred morphology affects the IR properties of spirals in different ways. These are well illustrated by the analysis in Huang et al. of a "combined" sample of IR bright, intermediate and faint populations, shown in Figures 2 and 3.

(1) Among galaxies with $L_{F I R} / L_{B}>0.33$, barred systems stand out strongly, both in relative IR luminosity and in mid-IR color, from unbarred galaxies: those with the most vigorous star formation may all be barred.

(2) Among the galaxies with $L_{F I R} / L_{B}<0.1$, barred systems have lower relative IR luminosity. Unfortunately these weak-IR galaxies have almost no 12 and $25 \mu \mathrm{m}$ IRAS detections, so use of mid-IR color diagnostics must await future observations, perhaps with ISO.

Different mechanisms are likely to explain the different effects of bars on galaxies in different IR-excess regimes. There is a good correlation of $L_{F I R} / L_{B}$ with $L_{F I R}$ in the combined sample of Huang et al. (1995), but $N O$ correlation with $L_{B}$. This strongly implies that the effect of barredness is most apparent amongst those galaxies which are relatively the most IR-luminous, simply because those whose IR luminosity is currently being enhanced by their bars have thereby been boosted into the relatively IR-luminous group. Amongst the less IR-luminous systems of the midrange, the fractional increase in $L_{F I R}$ is insufficient to make a marked difference, but the mid-IR colors are still affected. The faintest systems in the IR, as suggested by Isobe \& Feigelson, may be galaxies whose starbursts have subsided, but whose stellar populations have not yet had time to resupply enough new ISM for the thermalization of the background stellar radiation field or for the bar to re-initiate the starburst cycle, if indeed there is one - see below.

Two major uncertainties remain. Are bars necessary for the occurrence of starbursts in undisturbed spirals, as suggested by Hawarden et al. (1986b) and the statistics of the IR-luminous samples of Pompea \& Rieke (1990) and of Young \& Devereux (1991)? For while the four SA systems with SB-like IR properties in these three samples may just be misclassified, Figure 3, despite the dramatic difference between the distributions of SA and SB galaxies, contains $~$ 10 SAs with SB-like colors: misclassification on this scale appears unlikely. The second uncertainty lies in the strength of the bar required to have an effect on 


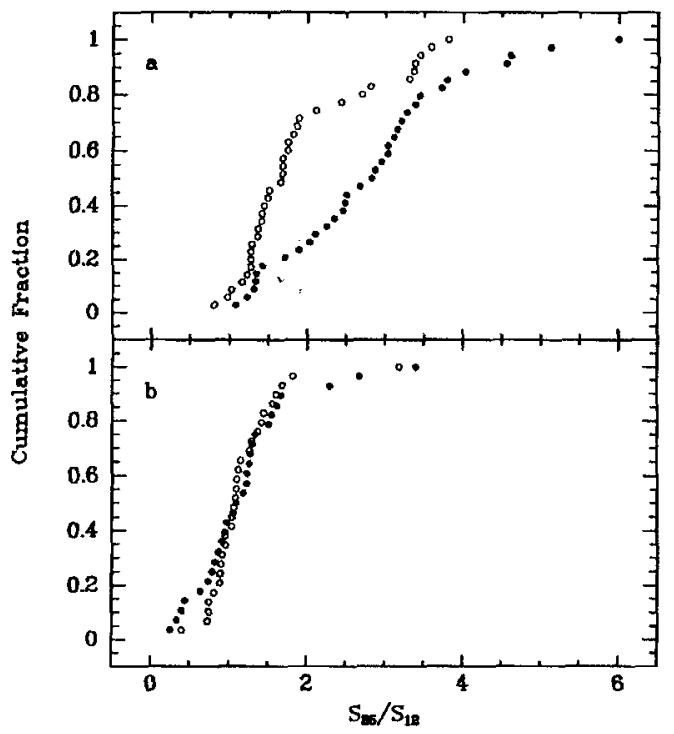

Figure 3. Cumulative distributions of the mid-IR 25/12 flux ratio for the early-type galaxies in the combined sample of Huang et al. (a) for galaxies with $L_{F I R} / L_{B}$ above, and (b) below, 1/3. Note the dramatically different distributions of SA and SB systems, but also that a number of SAs have high 25/12 values.

the IR properties of a system. Here the data are contradictory: Hawarden et al. $(1986 \mathrm{a}, \mathrm{b})$ and Devereux (1987) clearly include SAB galaxies with starburst-like colors, but SABs are indistinguishable from SAs in all samples in Huang et al. (1995).

Our picture is only part of a much more complex structure, not yet assembled: a self-consistent scenario of the genesis and evolution of bars in spiral galaxies and their effects on their parent systems. For example, others at this meeting review the intriguing implications of the instability of bars in the presence of a central mass concentration, on one hand, and on the other their evident efficiency at collecting ISM, a fair fraction of which must remained trapped near the nucleus as stellar and substellar remnants, building just such a concentration. Understanding the origins and incidence (duty cycles? one offs?) of strong IR emission from barred spirals is likely to be a critical step in the assembly of the abovementioned scenario.

\section{References}

Athanassoula, E. 1992a, MNRAS, 259, 328

Athanassoula, E. 1992b, MNRAS, 259, 345 
de Jong, T., Clegg, P. E., Soifer, B. T., Rowan-Robinson, M., Habing, H. J., Houck, J. R., Aumann, H. H. et al. 1984, ApJ, 278, L67

de Vaucouleurs, G. \& de Vaucouleurs, A. 1964. Reference Catalog of Bright Galaxies, Austin: University of Texas Press.

de Vaucouleurs, G., de Vaucouleurs, A., \& Corwin, H. G., Jr. 1976. Second Reference Catalog of Bright Galaxies, Austin, Texas: The University of Texas Press.

Devereux, N. A., Becklin, E. E., \& Scoville, N. 1987, ApJ, 312, 529

Devereux, N. A. 1987, ApJ, 323, 91

Dressel, L. L. 1988, ApJ, 329, L69

Hawarden, T. G., Fairclough, J. H., Joseph, R. D., Leggett, S. K., \& Mountain, C. M. 1986a, in Light on Dark Matter, F. P. Israel, Dordrecht: Reidel 1986,455

Hawarden, T. G., Mountain, C. M., Leggett, S. K., \& Puxley, P. J. 1986b, MNRAS, 221, 41p

Huang, J. H., Gu, Q. S., Su, H. J., Hawarden, T. G., Liao, X. H. \& Wu, G. X. 1995, A\&A, in press

Hummel, E. 1981, A\&A, 93, 93

Isobe, T. \& Feigelson, E. D. 1992, ApJS, 79, 197

Lonsdale, C. J., Persson, S. E., \& Matthews, K. 1984, ApJ, 287, 95

Pompea, S. M. \& Rieke, G. H. 1990, ApJ, 356, 416

Puxley, P. J., Hawarden, T. G., \& Mountain, C. M. 1988, MNRAS, 231, 465

Roberts, W. W., Huntley, J. M., \& van Albada, G. D. 1979, ApJ, 233, 67

Rowan-Robinson, M. \& Crawford, J. 1988, MNRAS, 238, 523

Sandage, A. \& Tammann, G. A. 1981, A Revised Shapley-Ames Catalog of Bright Galaxies, Carnegie Institute, Washington, DC

Sanders, D. B., Soifer, B. T., Elias, J. H., Madore, B. F., Matthews K., Neugebauer G., \& Scoville, N. Z. 1988, ApJ, 325, 74

Sérsic, J. L. \& Pastoriza, M. 1965, PASP, 77, 287

Sérsic, J. L. \& Pastoriza, M. 1967, PASP, 79, 152

Soifer, B. T., Boehmer, L., Neugebauer, G., \& Sanders, D. B. 1989, AJ, 98, 766

Schwarz, M. P. 1984, MNRAS, 209, 93

Telesco, C. M. \& Gatley, I. 1981, ApJ, 247, L11

Telesco, C. M. \& Gatley, I. 1984, ApJ, 284, 557

Telesco, C. M., Dressel, L. L., \& Wolstencroft, R. D. 1993, ApJ, 414, 120

Tubbs, A. D. 1982, ApJ, 255, 458

Véron-Cetty, M. P. \& Véron, P. 1993, ESO Scientific Report No.13

Young, J. S. \& Devereux, N. A. 1991, ApJ, 373, 414 